\title{
UV photometry of $\lambda$ Bootis stars and related objects
}

\author{
Ernst Paunzen ${ }^{1}$ and Ulrike Heiter ${ }^{2}$ \\ ${ }^{1}$ Institute for Astronomy, University Vienna, Türkenschanzstr. 17, A-1180 Vienna, Austria \\ email: Ernst.Paunzen@univie.ac.at \\ ${ }^{2}$ Department of Astronomy and Space Physics, Uppsala University, Box 515, SE-75120 \\ Uppsala, Sweden \\ email: ulrike@astro.uu.se
}

\begin{abstract}
We have compiled all available measurements in the UV region for the group of $\lambda$ Bootis stars. These data are compared to those of F-weak stars and known spectroscopic binary systems. We also use synthetic photometric values from standard atmospheric models to comment on the proposed spectroscopic binary nature of members of the $\lambda$ Bootis group which can be found in the literature.
\end{abstract}

Keywords. Stars: abundances, stars: chemically peculiar, stars: fundamental parameters

\section{Introduction}

The group of $\lambda$ Bootis stars comprise of true Population I, late B to early F-type stars, with moderate to extreme (up to a factor 100) surface underabundances of most Fe-peak elements and solar abundances of lighter elements $(\mathrm{C}, \mathrm{N}, \mathrm{O}$, and $\mathrm{S})$. This pattern is very similar to that of the interstellar medium. Only a maximum of about $2 \%$ of all objects in the relevant spectral domain are found to be $\lambda$ Bootis type stars.

\section{History}

The history of UV-observations for $\lambda$ Bootis stars began with the TD1 S2/68 experiment described by Boksenberg et al. (1973). Cucchiaro et al. (1980) already recognized several features (e.g. depression at $1600 \AA$ ) which seem typical for the $\lambda$ Bootis group at a very low resolution. They gave a list of seven bona-fide $\lambda$ Bootis candidates. But it turned out that the resolution was too poor to unambiguously identify $\lambda$ Bootis stars.

Baschek et al. (1984) were the first who used low and high resolution IUE-spectra to distinguish $\lambda$ Bootis from normal type stars. They concluded that carbon (and possibly nitrogen) might not share the underabundances of the heavier elements. The two main criteria to identify true $\lambda$ Bootis stars, the strong depressions at $1600 \AA$ and $3040 \AA$ were also detected in normal type, FHB and cool DA white dwarfs by Jaschek et al. (1985). Holweger et al. (1994) later explained the $1600 \AA$ feature: it is caused by quasimolecular absorption leading to a satellite in the Lyman $\alpha$ profile due to perturbations by neutral hydrogen.

As a further step, Baschek \& Slettebak (1988) made the first qualitative UV abundance analysis of ten suspected $\lambda$ Bootis stars. The result was that the light elements $(\mathrm{C}, \mathrm{N}$ and $\mathrm{O}$ ) are slightly overabundant whereas the heavier elements ( $\mathrm{Mg}, \mathrm{Al}, \mathrm{Si}, \mathrm{Mn}, \mathrm{Fe}$ and $\mathrm{Ni})$ are moderately deficient $(\simeq 0.5 \mathrm{dex})$. It should be noted that no clear conclusion for sulphur was derived.

As a summary of all previous efforts, Faraggiana et al. (1990) tried to establish criteria 
for the definition of a homogeneous group of $\lambda$ Bootis stars (only the relevant characteristics deduced in the UV region are listed):

- The ratio $\mathrm{CI} / \mathrm{Al}$ II should be greater than 1.5

- The bump at $1600 \AA$ should be detectable

- The discrepancy between the MK and UV type should be greater than two spectral subclasses

\section{IUE data}

With the creation of the IUE Final Archive in 1998 it was possible to access all IUE images which are uniformly processed and calibrated, thus making a homogeneous analysis possible.

Solano \& Paunzen (1998) and Solano \& Paunzen (1999) presented the results from a careful analysis of the IUE final archive using the low and high resolution spectra separately. Their goal was to establish criteria in the UV region without using data from the optical domain and to unambiguously distinguish $\lambda$ Bootis from FHB stars.

From the low resolution spectra they concluded that a star is considered to be a member of the $\lambda$ Bootis group if:

- C I $1657 / \mathrm{Al}$ II $1670>2.0$

- C I $1657 / \mathrm{Ni}$ II $1741>2.5$

These limits have been set in such a way that they are valid for the entire range of effective temperatures. Small uncertainties due to variations in $\log g$ and $[\mathrm{M} / \mathrm{H}]$ have also been taken into account to make these limits independent of physical parameters such as the effective temperature and surface gravity. Moreover, they are also independent of the evolutionary status since a clear distinction between $\lambda$ Bootis and FHB stars was established. From the analysis of the high resolution spectra they were able to add the following criteria for a star being a member of the $\lambda$ Bootis group:

- C I 1657/Si II $1527>3.0$

- C I $1657 /$ Ca II $1839>8.0$

The four listed criteria in the UV region are unambiguous and do reflect the typical abundance pattern for this group as listed in Heiter (2002).

\section{TD1 data}

Gerbaldi et al. (2003) used the TD1 photometry to search for undetected spectroscopic binary systems among members of the $\lambda$ Bootis group. From the list of Paunzen et al. (2002), 36 stars have been included. From these, twenty two were found to fulfill the criteria of a membership, the flux of additional ten objects could not be fitted with standard ATLAS9 models and four stars were found to have apparent solar elemental abundances. For three of the latter (HD 74873, HD 170680 and HD 221756), Paunzen et al. (2002) list the following iron abundances relative to the solar values: $-0.7,-0.5$ and -0.59 dex, respectively. This might indicate that the fitting of theoretical spectra to observations assuming a fixed effective temperature and surface gravity for an individual object is insufficient and erratic in some cases. We have investigated the TD1 magnitudes of the F-weak objects taken from Hauck et al. (1991), $\lambda$ Bootis stars as well as known spectroscopic binary systems (taken from the Eighth Catalogue of the Orbital Elements of Spectroscopic Binary Systems). Figure 1 shows the result from which we conclude that:

- F-weak type stars can be easily distinguished from $\lambda$ Bootis type objects 


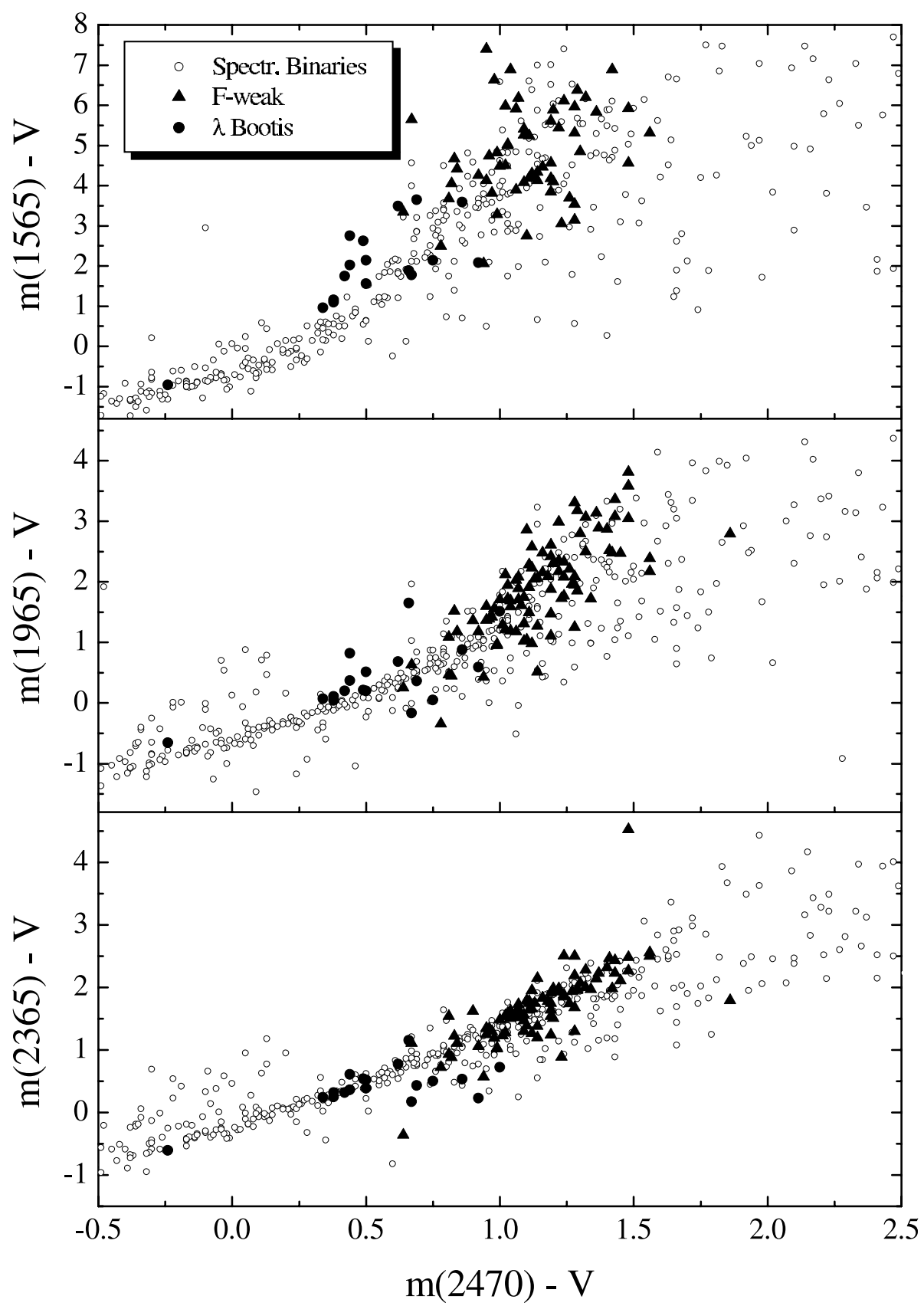

Figure 1. TD1 observations for known spectroscopic binary systems, F-weak and $\lambda$ Bootis stars

- In the passbands of $1565 \AA$ and $1965 \AA$, the SBs systems seem to occupy a different region than the members of the $\lambda$ Bootis group

- Still there is a significant overlap of SB and $\lambda$ Bootis type objects 
As next step, we will compare synthesized TD1 magnitudes with observed ones to check the existing calibrations.

\section{Acknowledgements}

This work benefitted from the Fonds zur Förderung der wissenschaftlichen Forschung, project (S7303-AST and P14984).

\section{References}

Baschek, B., Slettebak, A. 1988, A\&SA 207, 112

Baschek, B., Heck, A., Jaschek, C., et al. 1984, A\&SA 131, 378

Boksenberg, A., Evans, R.G., Fowler, R.G., et al. 1973, MNRAS 163, 291

Cucchiaro, A., Jaschek, M., Jaschek, C., Macau-Hercot, D. 1980, A\&AS 40, 207

Faraggiana, R., Gerbaldi, M., Böhm, C. 1990, A\&A 235, 311

Gerbaldi, M., Faraggiana, R., Lai, O. 2003, A\& A 412, 447

Hauck, B., Jaschek, C., Jaschek, M., Andrillat, Y. 1991, A\&A 252, 260

Heiter, U. 2002, A\& $A$ 381, 959

Holweger, H., Koester, D., Allard, N.F. 1994, A\&\&A 290, L21

Jaschek, M., Baschek, B., Jaschek, C, Heck, A. 1985, A 6 A 152, 439

Paunzen, E., Iliev, I.Kh., Kamp, I., Barzova, I.S. 2002, MNRAS 336, 1030

Solano, E., Paunzen, E. 1998, A\&A 331, 633

Solano, E., Paunzen, E. 1999, A\&A 348, 825 